\title{
ULTRAFAST ENERGY RELAXATION AND EXCITATION DELOCALIZATION IN EXCITED STATES OF ZINC PORPHYRIN DIMERS AND TRIMER
}

\author{
I. YamaZaKi*, S. Akimoto, T. Yamazaki \\ Graduate School of Molecular Chemistry, Faculty of Engineering, Hokkaido University \\ Sapporo 060-8628, Japan \\ H. Shiratori and A. Osuka \\ Graduate School of Chemistry, Kyoto University, Sakyo-ku, Kyoto 606-8224, Japan
}

\begin{abstract}
Ultrafast excited-state relaxation process has been studied with zinc porphyrin dimers and circular trimer. Following $80 \mathrm{fs}$ excitation at Soret band $(420 \mathrm{~nm})$ or $Q$ band $(580 \mathrm{~nm})$ of zinc porphyrin, the fluorescence decay curves exhibit ultrafast decays with lifetimes of $80 \mathrm{fs}$ in o-dimer, $450 \mathrm{fs}$ in trimer and $540 \mathrm{fs}$ in $m$-dimer. The time-resolved fluorescence spectra show that the fast decay process correspond to disappearance of monomer-like emission followed by red-shifted and broaden spectra. These ultrafast processes are assigned as due to excitation transfer among monomers and delocalization of excitation yielding excitonic states.
\end{abstract}

PACS numbers: $31.50 .+\mathrm{w}, 78.47 .+\mathrm{p}$

\section{Introduction}

Photophysical processes such as excitation energy transfer and electron transfer are expected to occur very fast in organized molecular systems in which reactant molecules are arranged with close proximity and optimal orientation, and they can be coupled to an adjacent molecule with relatively strong molecular interaction. In 1960's, Förster [1,2] formulated the excitation energy transfer under the dipole-dipole resonance interaction, and classified the energy transfer into three cases, strong coupling case, medium coupling case and very-weak coupling case, depending on magnitude of the interaction energy and transfer rate. Subsequently, Kenkre and Knox [3] presented a generalized formulation for the excitation transfer rate. Most of previous studies are concerned with the very-weak coupling case, in which the energy transfer occurs irreversibly in timescale of longer than $10 \mathrm{ps}$. The

\footnotetext{
*corresponding author; e-mail: yamiw@eng.hokudai.ac.jp, fax: 81-11-709-2037
} 
strong coupling case appears in the Frenkel exciton of molecular crystals, where the transfer occurs extremely fast and the excitation is instantaneously delocalized as a whole through the molecular system within an excitation pulse width of $\sim 1 \mathrm{ps}$. In the case of medium coupling case, the transfer occurs in 1-0.1 ps under the interaction energy of $10^{2}-10^{3} \mathrm{~cm}^{-1}$. One can expect this condition to be met in the organized molecular system.

On the other hand, it has been recognized for long time that the intramolecular processes like internal conversion, vibrational relaxation (VR), and internal vibrational redistribution (IVR) are extremely fast in condensed matters like solution and solid states [4]. Very few is known for the kinetic parameter of these intramolecular processes. Recent experiments on time-resolved spectroscopies for excited molecules in solution, however, have revealed that VR occurs in timescales of the order of 1-10 ps, and IVR occurs in the order of $100 \mathrm{fs}$ [5-7]. Note that one can prepare the organized molecular system in which the reaction can occur less than 1 ps under relatively strong molecular interaction. Then the excitation transfer can compete with the VR and/or IVR processes, and it becomes involving a direct process from highly excited vibrational state initially photoexcited $[8,9]$. Moreover if the reaction rate is comparable to or exceeding the energy dissipation with environments, one can observe this reaction as coherent processes accompanying quantum beat phenomena. Recent studies on photochemical processes in highly-organized molecular systems of natural photosynthetic reaction centers have demonstrated striking features of non-equilibrium reactions and coherent processes, in which an exciton propagates as a quantum wave packet through a molecular channel.

We have investigated ultrafast excitation migration and relaxation in circular arrangement of zinc porphyrin ( $\mathrm{ZnP}$ ) dimers and trimer, which are bridged by a phenyl ring, with a femtosecond fluorescence up-conversion method. The structures of molecules concerned here are shown in Fig. 1. Because of close proximity of $\mathrm{ZnP}$ rings, excitonic states are formed under a strong interaction around the Soret band and probably a weak interaction around the $Q$ band. The time-resolved fluorescence spectra revealed excitation delocalization in timescale less than 1 ps.

\section{Experimental}

The organized molecular systems of circular arrangement of $\mathrm{ZnP}$ rings were synthesized with a method described in previous papers [10,11]. The molecules concerned in this study are monomer, $o$-, $m$ - and $p$-dimers and trimer (Fig. 1).

The time-resolved fluorescence measurements were made with an up-conversion method [12,13] using a Ti:sapphire laser (Spectra Physics, Tsunami, $840 \mathrm{~nm}, 80 \mathrm{MHz}$ ) which was pumped with a diode-pumped solid state laser (Spectra Physics, Millennia X). The fundamental pulses are separated into two beams; the one is frequency-doubled by a BBO crystal $(420 \mathrm{~nm})$ and is used to excite the sample, and the other beam serves as a gate pulse. The gate pulse traverses a variable optical delay of $2 \mu \mathrm{m} / \mathrm{step}(6.7 \mathrm{fs})$, while the excitation pulse traverses a fixed delay before being focused into a $1 \mathrm{~mm}$ sample cell. The fluorescence emission and the gate pulse are focused into a BBO crystal of $0.5 \mathrm{~mm}$ thickness in a type-1 phase matching geometry. To avoid polarization effects, the angle between 

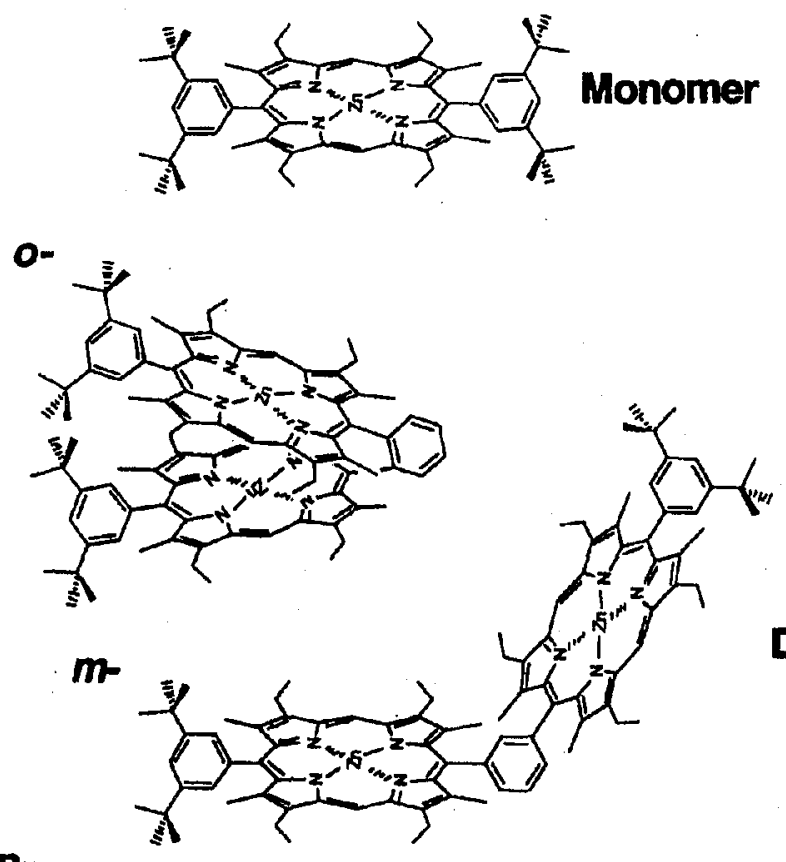

Dimers
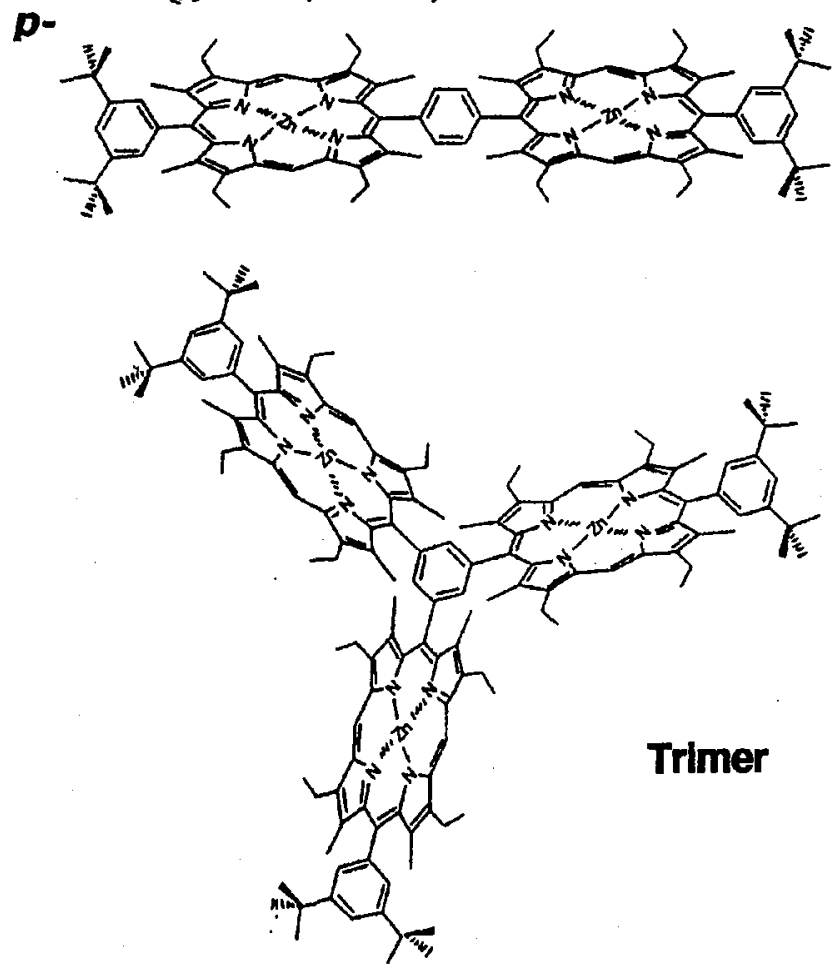

Fig. 1. Molecular arrangements of zinc porphyrin ( $\mathrm{ZnP})$ monomer, $o-, m$ - and $p$-dimers and trimer concerned in the present study. 
the polarizations of excitation and probe beams were set to the magic angle with a $\lambda / 2$ plate. The sum-frequency signal of the Raman scattering light from benzene liquid and the gate pulse yields an instrumental response function of $160 \mathrm{fs}$ pulse width (FWHM). All measurements were carried out at room temperature.

\section{Results}

\subsection{Absorption and fluorescence spectra}

Figures 2 and 3 show absorption and fluorescence spectra of $\mathrm{ZnP}$ monomer, three dimers and trimer in benzene or THF (in o-dimer) solution. The absorption spectra consist of strong Soret band around 400-430 nm and much weaker $Q$ band around $530-580 \mathrm{~nm}$ which are the spectra typical of zinc porphyrin. It is seen in Fig. 2 that the Soret band undergoes a significant change in going from monomer to trimer, i.e., red shift and band splitting, while the $Q$ band exhibit only a small red shift with the band profiles being constant. The fluorescence spectrum also changes with respect to band position and band profile, corresponding to changes in the absorption spectrum. The wavelengths of band maxima are summarized in Table I.

\section{TABLE I}

Wavelengths (in $\mathrm{nm}$ ) of band maxima in absorption and fluorescence spectra of $\mathrm{ZnP}$ monomer, dimers and trimer in benzene and THF (in o-dimer) solution.

\begin{tabular}{|c|c|c|c|c|c|}
\hline \multirow{3}{*}{ Bands } & \multicolumn{5}{|c|}{ Compounds } \\
\hline & \multirow[t]{2}{*}{ Monomer } & \multicolumn{3}{|c|}{ Dimers } & \multirow[t]{2}{*}{ Trimer } \\
\hline & & $0-$ & $m-$ & $p-$ & \\
\hline \multirow{3}{*}{$\begin{array}{l}\text { Absorption } \\
\text { Soret band }\end{array}$} & \multirow[t]{3}{*}{412} & \multirow[t]{3}{*}{400} & 410 & \multirow[t]{3}{*}{425} & 414 \\
\hline & & & 427 & & 428 \\
\hline & & & & & 444 \\
\hline Absorption & 539 & 545 & 543 & 543 & 549 \\
\hline \multirow[t]{3}{*}{$Q$ band } & \multirow[t]{3}{*}{573} & 562 & 575 & 575 & 576 \\
\hline & & 578 & 587 & & 593 \\
\hline & & 612 & & & \\
\hline \multirow[t]{2}{*}{ Fluorescence } & 580 & 653 & 597 & 587 & 608 \\
\hline & 633 & 701 & 645 & 642 & 653 \\
\hline
\end{tabular}

Among the molecules, $o$-dimer exhibits unusual spectra; a very diffuse absorption band extends to further longer wavelength ( $612 \mathrm{~nm}$ in $Q$ band) and the fluorescence spectrum start to rise at much longer wavelength $(610 \mathrm{~nm})$. This may suggest that the two $\mathrm{ZnP}$ rings interact strongly one another. 


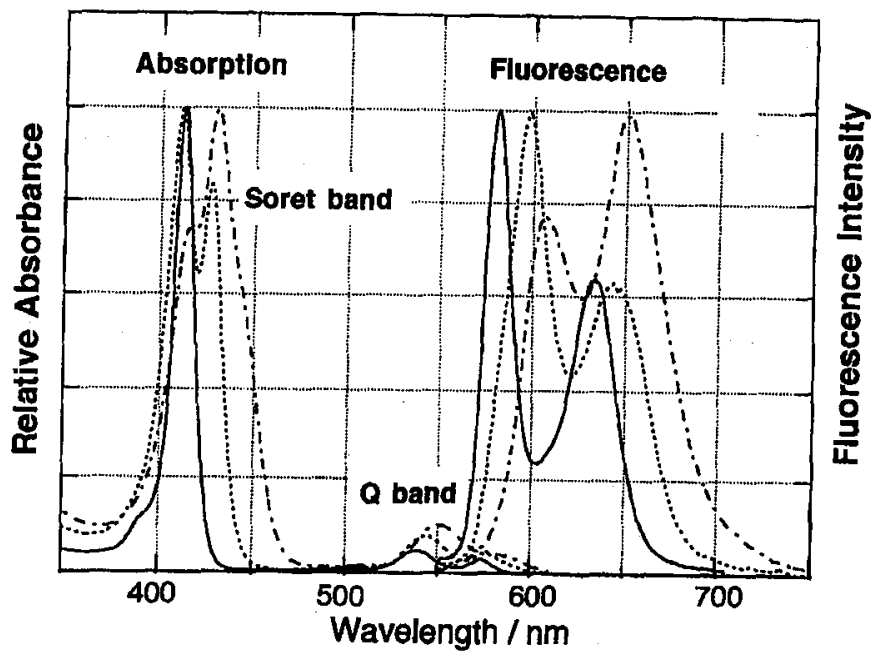

Fig. 2. Absorption and fluorescence spectra of $\mathrm{ZnP}$ monomer, $m$-dimer and trimer in benzene solution.

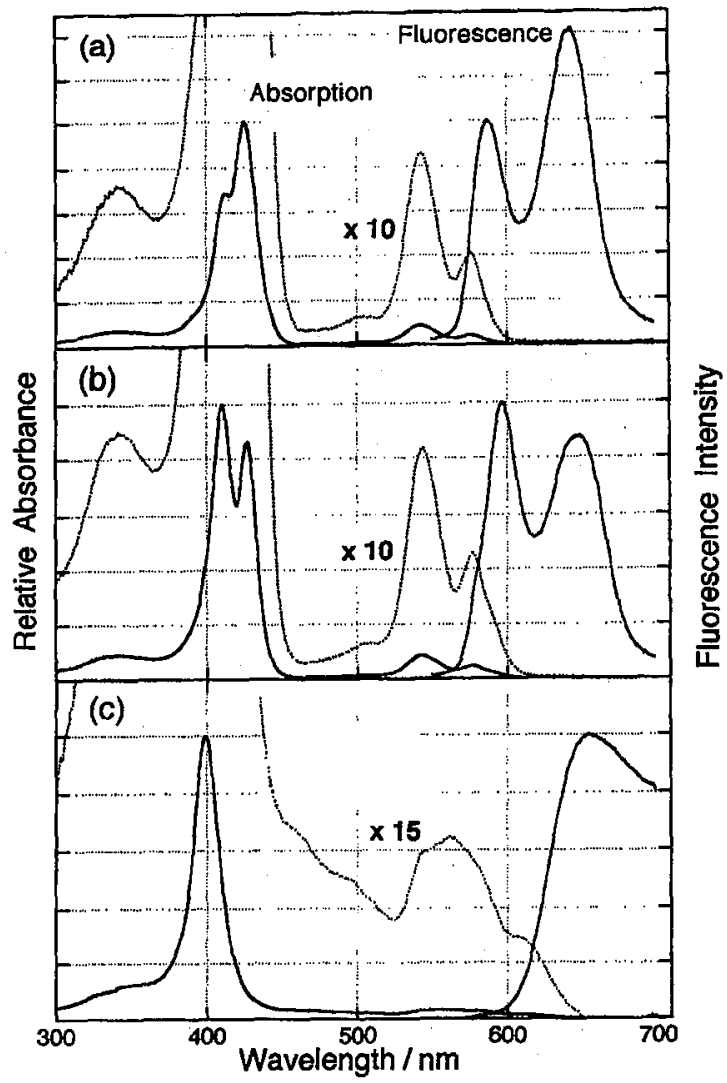

Fig. 3. Absorption and fluorescence spectra of three dimers; (a) $p$-dimer, (b) $m$-dimer and (c) o-dimer. 


\subsection{Theoretical calculation of exciton splitting}

To investigate the absorption change among monomer, dimers and trimer, we calculated excitonic level structures by using the exciton coupling model [14-16]. We assume the molecular geometry of trimer by referring to a previous study by Eriksson et al. [17] that the trimer has $C_{3}$ symmetry and for the spatial orientation of $\mathrm{ZnP}$ rings respective rings are tilted by $20^{\circ}$ to the $C_{3}$ symmetry axis, as shown in Fig. 4. Let the wave functions of the ground state and the electronically excited

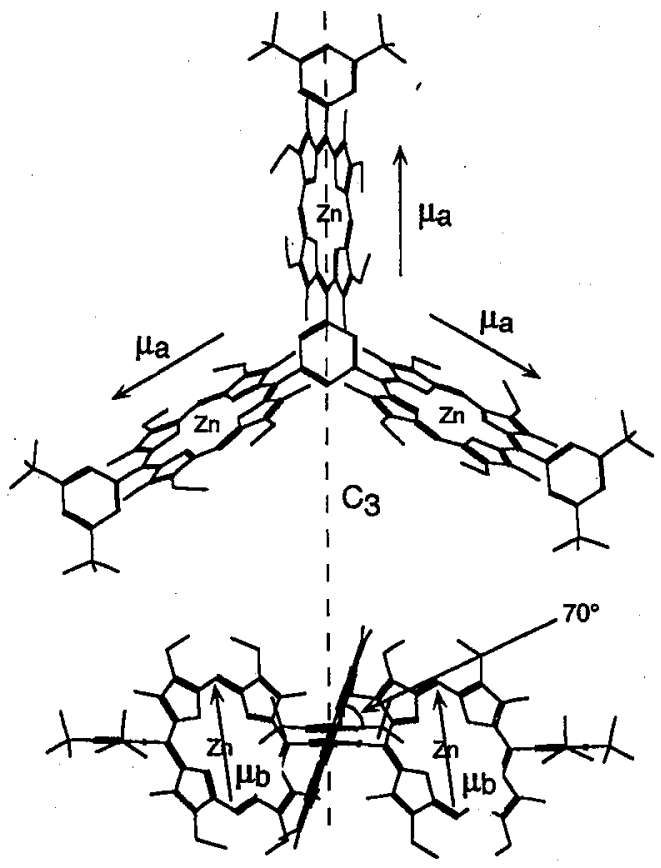

Fig. 4. Molecular structure of $\mathrm{ZnP}$ trimer (top view and side view). Three $\mathrm{ZnP}$ rings are arranged with $C_{3}$ symmetry. The center-to-center distance between neighboring $\mathrm{ZnP}$ rings is $11 \AA$. The angle of $\mathrm{ZnP}$ ring is $70^{\circ}$ to the molecular plane of phenyl ring. The two nearly degenerate electronic transitions are shown in their transition dipole moments.

state of $i$-th $\mathrm{ZnP}$ ring denote as $\phi_{i}$ and $\phi_{i}^{*}$, respectively. In the presence of a Coulombic interaction $V_{i j}$ between $\mathrm{ZnP}$ rings $i$ and $j$, the electronic Hamiltonian of the molecular system is expressed as

$$
H=\sum_{i=1}^{N} H_{i}+\sum_{i=1}^{N} \sum_{j>i}^{N} V_{i j},
$$

where $H_{i}$ is the Hamiltonian of $i$-th $\mathrm{ZnP}$ ring. Then the wave function and the energy of singly excited states can be obtained by solving an eigenvalue problem, as follows: for the dimer,

$$
\Phi_{1}=\frac{1}{\sqrt{2}}\left(\phi_{1}^{*} \phi_{2}+\phi_{1} \phi_{2}^{*}\right), \quad E_{1}=\alpha+\beta,
$$




$$
\Phi_{2}=\frac{1}{\sqrt{2}}\left(\phi_{1}^{*} \phi_{2}-\phi_{1} \phi_{2}^{*}\right), \quad E_{2}=\alpha-\beta,
$$

where $\alpha$ is the energy of configuration $\phi_{1}^{*} \phi_{2}$ or $\phi_{1} \phi_{2}^{*}$, and $\beta$ is the resonance integral $\left\langle\phi_{1}^{*} \phi_{2}\left|V_{12}\right| \phi_{1} \phi_{2}^{*}\right\rangle$ which can be expanded into point multipole series and usually taken as an approximate form of dipole-dipole term. $\beta$ represents the interaction between the transition dipole moments $\mu_{1}$ and $\mu_{2}$ of both $\mathrm{ZnP}$ rings,

$$
\beta \propto \frac{\left|\mu_{1}\right|\left|\mu_{2}\right|}{r^{3}}\left(\cos \alpha+3 \cos \theta_{1} \cos \theta_{2}\right),
$$

where $r$ is the center-to-center distance between adjacent porphyrin rings, and $\alpha, \theta_{1}$ and $\theta_{2}$ are angles between $\mu_{1}$ and $\mu_{2}$. The square of $\beta$ is proportional to the oscillator strength of the transitions between the ground and excited states of respective $\mathrm{ZnP}$ rings. Similarly, we obtain the wave function and the energy for the trimer,

$$
\begin{array}{ll}
\Phi_{1} & =\frac{1}{\sqrt{3}}\left(\phi_{1}^{*} \phi_{2} \phi_{3}+\phi_{1} \phi_{2}^{*} \phi_{3}+\phi_{1} \phi_{2} \phi_{3}^{*}\right), \quad E_{1}=\alpha+2 \beta, \\
\Phi_{2} & =\frac{1}{\sqrt{2}}\left(\phi_{2}^{*} \phi_{3}-\phi_{2} \phi_{3}^{*}\right), \quad E_{2}=\alpha-\beta, \\
\Phi_{3}=\frac{1}{\sqrt{6}}\left(2 \phi_{1}^{*} \phi_{2} \phi_{3}-\phi_{1} \phi_{2}^{*} \phi_{3}-\phi_{1} \phi_{2} \phi_{3}^{*}\right), \quad E_{3}=\alpha-\beta .
\end{array}
$$

We calculated the energy levels of exciton states and their oscillator strengths of the absorption (Soret band) transition for the two nearly degenerate excited states of $\mathrm{ZnP}$ monomer and their optical transition dipole moments $\mu_{a}$ and $\mu_{b}$. The results are summarized in Table II and are illustrated in Fig. 5 in which
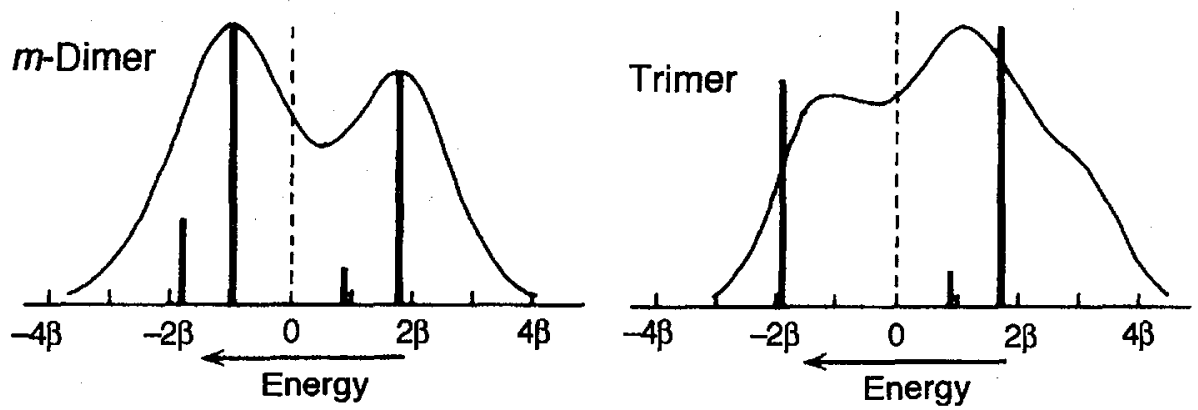

Fig. 5. Calculated spectra of excitonic states of Soret band compared with the experimental absorption spectra. The coordinates are taken in unit of the interaction energy $\beta$. In this figure, we took $\beta=400 \mathrm{~cm}^{-1}$. The length of vertical lines shows magnitude of the oscillator strength taken as summation of the contribution from $\mu_{a}$ and $\mu_{b}$ (Table II). 
TABLE II

Results of exciton splitting calculation; the energy levels and the oscillator strengths for absorption transitions.

\begin{tabular}{lc|c|c|c|c|c|c}
\hline \hline \multirow{2}{*}{ Exciton states $^{a}$} & \multirow{2}{*}{$\begin{array}{c}\text { Energy } \\
\end{array}$} & $E$ & $\begin{array}{c}\text { Dipole } \\
\text { moment }^{c}\end{array}$ & \multicolumn{2}{|c|}{$\mu_{b}^{d}$} & \multicolumn{2}{|c}{$\mu_{a}^{d}$} \\
\cline { 5 - 8 } & & $\mu$ & $\Delta E^{e}$ & $f_{b}^{f}$ & $\Delta E^{e}$ & $f_{a}^{f}$ \\
\hline Dimer & & & & & & \\
& $\Phi_{1}$ & $\alpha+\beta$ & $(1 / \sqrt{2})\left(\mu_{1}+\mu_{2}\right)$ & -0.91 & 1.82 & -1.75 & 0.5 \\
& $\Phi_{2}$ & $\alpha-\beta$ & $(1 / \sqrt{2})\left(\mu_{1}-\mu_{2}\right)$ & 0.91 & 0.18 & 1.75 & 1.5 \\
\hline Trimer & & & & & & & \\
& $\Phi_{1}$ & $\alpha+2 \beta$ & $(1 / \sqrt{3})\left(\mu_{1}+\mu_{2}+\mu_{3}\right)$ & -1.82 & 2.60 & -3.50 & 0 \\
& $\Phi_{2}$ & $\alpha-\beta$ & $(1 / \sqrt{2})\left(\mu_{2}-\mu_{3}\right)$ & 0.91 & 0.18 & 1.75 & 1.5 \\
& $\Phi_{3}$ & $\alpha-\beta$ & $(1 / \sqrt{6})\left(2 \mu_{1}-\mu_{2}-\mu_{3}\right)$ & 0.91 & 0.18 & 1.75 & 1.5 \\
\hline
\end{tabular}

${ }^{a}$ Wave functions of the exciton state are described in the text.

${ }^{b}$ Energy of the exciton state represented with the energies of local excited state $\alpha$ and of the interaction energy $\beta$. The theoretical formulation of $\alpha$ and $\beta$ are given in the text.

${ }^{c}$ Electronic transition dipole moment of absorption from the ground state to the exciton state expressed in terms of sum of respective transition dipole moments of two or three $\mathrm{ZnP}$ rings.

${ }^{d}$ Transition dipole moment of one of the two nearly degenerate transition of ZnP ring.

${ }^{e}$ Band energies of the exciton state in a unit of the interaction energy $\beta$.

$f$ Relative oscillator strength.

the Soret bands spectra of $m$-dimer and trimer are superposed. It is seen that the calculated spectra are in approximately good agreement with the spectra for $m$-dimer and trimer when we take $\beta=400 \mathrm{~cm}^{-1}$. For the exciton splitting in $Q$-band, the similar fitting with the calculated spectra is rather difficult because of complexity of absorption band structure. It is expected, however, the exciton interaction to be much smaller than in the Soret band, since the transition dipole moment is small. In fact, the band profiles of $Q$ bands in dimers and trimer are basically quite the same as that of monomer.

A detailed analysis for the absorption band structure reveals that, in $o$ and $m$-dimers and trimer, other weak absorption bands are involved in the red edge of $Q$ band. The band positions of the weak absorption are presented in later discussion (Fig. 8). We assume here that the higher state is of local excited state likely to the lowest excited state of monomer, and the lower state is of excitonic state in which the excitation is delocalized through a small interaction energy of $\sim 100 \mathrm{~cm}^{-1}$ in $m$-dimer and trimer, but through a fairly large interaction energy of $\sim 300 \mathrm{~cm}^{-1}$ in o-dimer. 


\subsection{Fluorescence decays and time-resolved spectra}

Figure 6 shows the fluorescence decay curves in subpicosecond time region for monomer, $m$-dimer, $o$-dimer and trimer obtained by monitoring at several different wavelengths. In monomer (Fig. 6a), no fast decay is involved and it shows only a long decay with lifetimes of $1.5 \mathrm{~ns}$. The decay curve of $p$-dimer exhibits no fast decay, and essentially the same as that of monomer. On the other hand, $o$ - and $m$-dimers exhibit very short decay with lifetimes of 80 fs and $540 \mathrm{fs}$, respectively (Fig. $6 \mathrm{~d}$ and $\mathrm{b}$ ). Also the trimer exhibits a fast decay component with lifetime of $450 \mathrm{fs}$ (Fig. 6c). The decay curves depend on monitoring wavelength. In the case of $o$-dimer (Fig. 6d), a very short decay is dominated in a short wavelength region, while in a longer wavelength region, only a long decay is involved with a rise component corresponding to the sharp decay. This means that the spectral profile at earlier time is quite different from those at later time region. The values of fluorescence lifetime are summarized in Table III. In order to see such spectral change in detail, we rewrote the decay profiles in the form of time-resolved spectra as shown in Fig. 7.

TABLE III

Fluorescence lifetimes $(\tau)$ and their amplitudes $(A)$ of monomer, three dimers and trimer.

\begin{tabular}{|c|c|c|c|c|c|}
\hline \multicolumn{2}{|c|}{ Compounds } & \multicolumn{2}{|c|}{ Subpicosecond region [ps] } & \multicolumn{2}{|c|}{ Picosecond region [ns] } \\
\hline & & $\tau_{1}\left(A_{1}\right)$ & $\tau_{2}\left(A_{2}\right)$ & $\tau_{1}\left(A_{1}\right)$ & $\overline{\tau_{2}\left(A_{2}\right)}$ \\
\hline Mono & & none & none & $1.5(1.0)$ & \\
\hline \multicolumn{6}{|c|}{ Dimers } \\
\hline \multirow[t]{2}{*}{$o-$} & $(610 \mathrm{~nm})$ & $0.08(0.88)$ & $1.4(0.11)$ & $1.3(1.0)$ & \\
\hline & $(650 \mathrm{~nm})$ & $0.89(-0.32)$ & & $1.3(1.0)$ & \\
\hline \multirow[t]{2}{*}{$m-$} & $(580 \mathrm{~nm})$ & $0.54(0.64)$ & $2.9(0.24)$ & $1.2(1.0)$ & $4.5(0.01)$ \\
\hline & $(640 \mathrm{~nm})$ & $0.73(0.56)$ & $3.3(0.23)$ & $1.2(0.95)$ & $4.5(0.05)$ \\
\hline$p$ & & none & none & $1.5(1.0)$ & \\
\hline \multicolumn{6}{|c|}{ Trimer } \\
\hline & $(580 \mathrm{~nm})$ & $0.45(0.58)$ & $2.8(0.30)$ & $0.80(1.0)$ & $3.1(0.01)$ \\
\hline & $(640 \mathrm{~nm})$ & $0.60(0.61)$ & & $0.80(0.90)$ & $3.1(0.10)$ \\
\hline
\end{tabular}

It is seen that the fluorescence spectrum changes within $1 \mathrm{ps}$ in $m$-dimer and $1 \mathrm{ps}$ in trimer. In every case, the spectrum at earlier time is rather sharp, and peak position is different from the later spectra. Particularly in o-dimer, a sharp structured band appears in the very beginning, and then changes rapidly to a diffuse and red-shifted spectrum. The fluorescence spectra at 6-8 ps correspond to the respective spectra of stationary excitation, and the earlier time spectra are similar to the respective monomer spectra. 

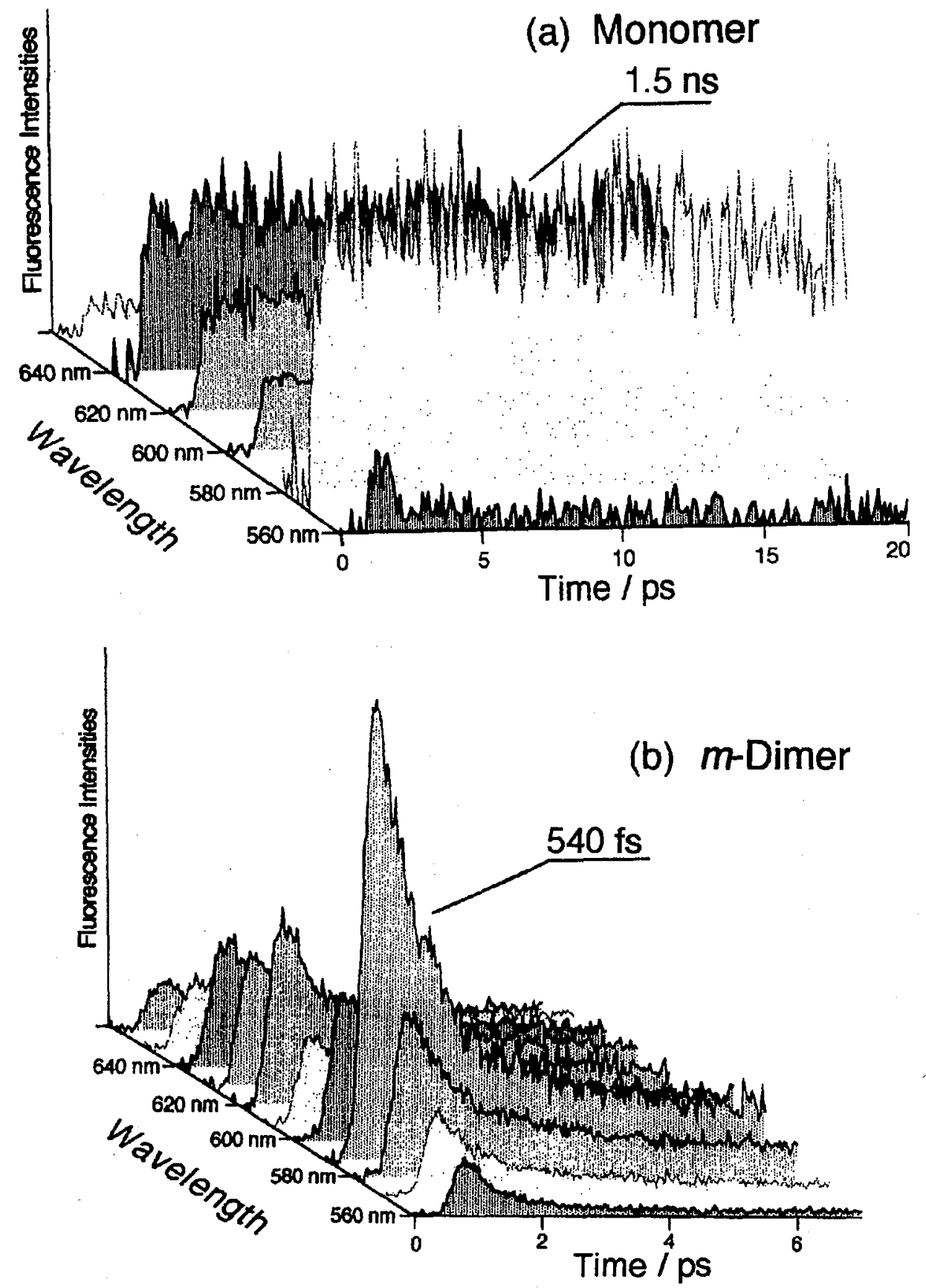

Fig. 6. Fluorescence decay curves of monomer (a) and $m$-dimer (b) monitored at different wavelengths. The excitation wavelength is $420 \mathrm{~nm}$. 

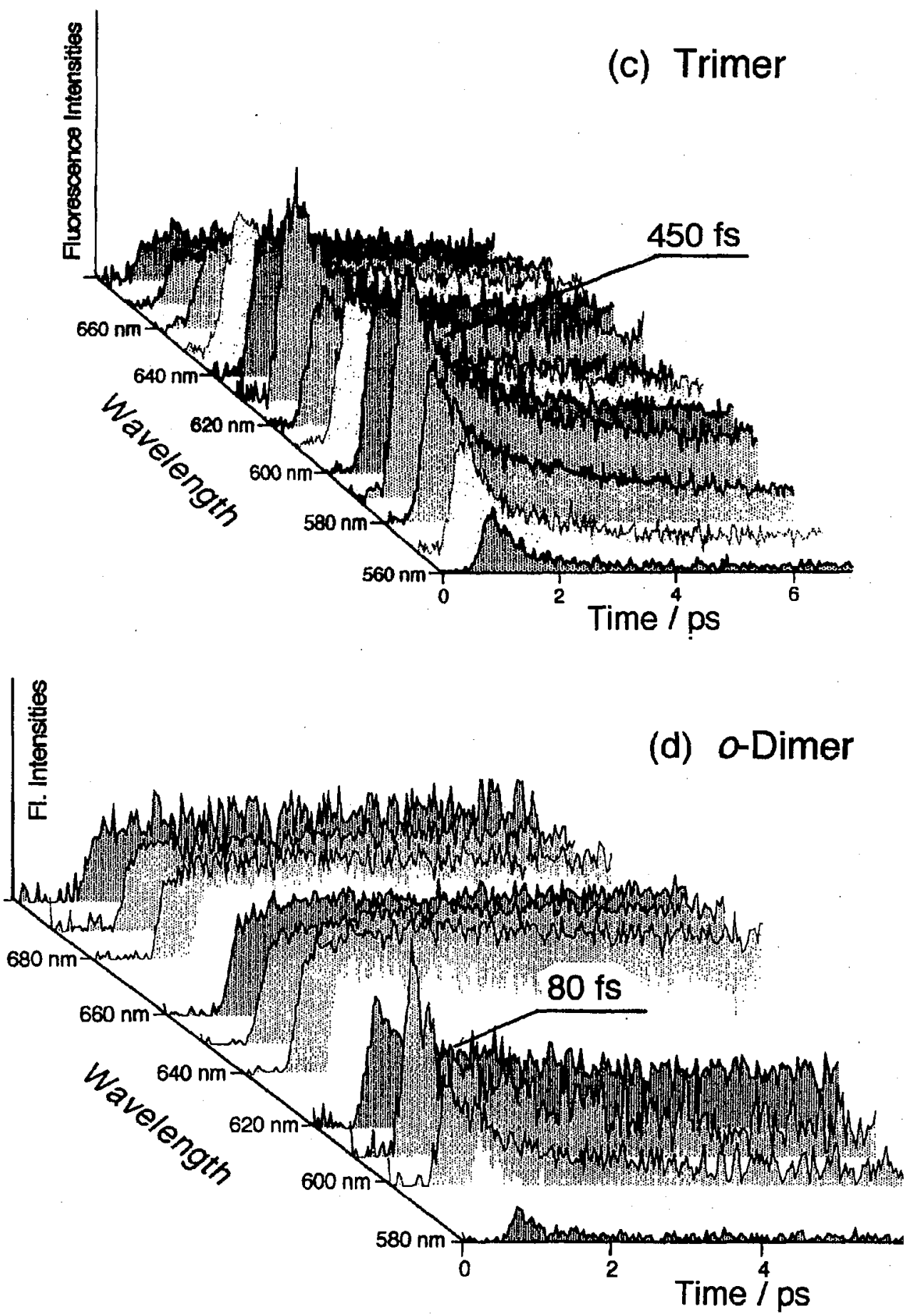

Fig. 6. Fluorescence decay curves of trimer (c) and o-dimer (d) monitored at different wavelengths. The excitation wavelength is $420 \mathrm{~nm}$. 
(a) m-Dimer

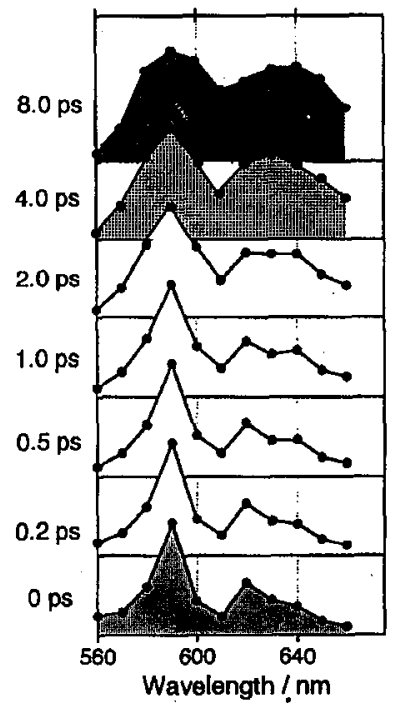

(b) Trimer

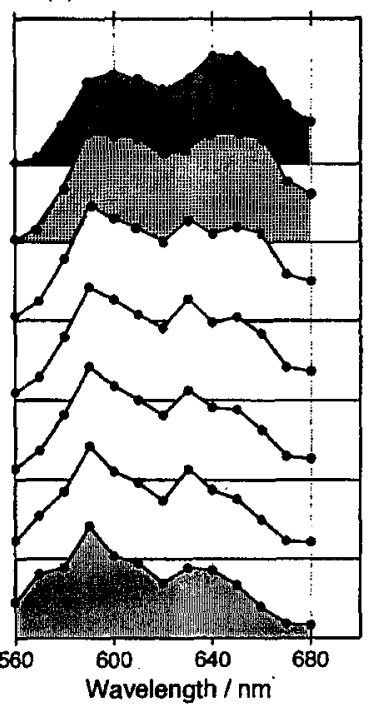

(c) o-Dimer

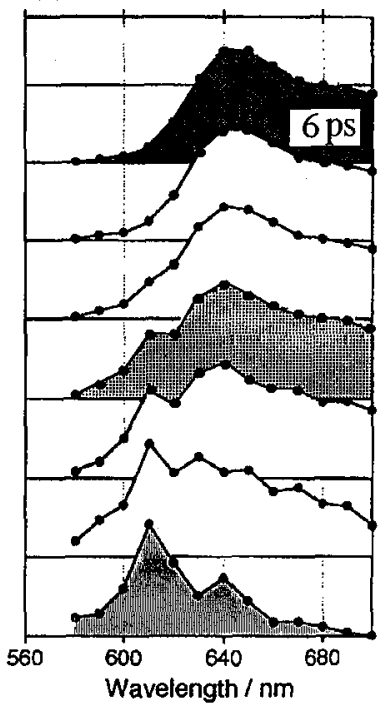

Fig. 7. Time-resolved fluorescence spectra of $m$-dimer (a), trimer (b) and $o$-dimer (c) obtained from the decay curves shown in Fig. 6. The spectra at 6.0 ps and $8.0 \mathrm{ps}$ are identical to the stationary-excitation fluorescence spectra.

These data were taken with laser excitation at Soret band (420 nm). Also we did experiment with excitation at $Q$ band $(580 \mathrm{~nm})$. Then we obtained similar fast fluorescence decays with corresponding lifetimes for dimers and trimer.

\section{Discussion}

The present experiment reveals that the excited states of $\mathrm{ZnP}$ dimers and trimer undergo subpicosecond relaxation, as well as nanosecond processes. The time-resolved fluorescence spectra show that the ultrafast relaxation processes are associated with changes in spectral profiles in time region of less than 1 ps. Here we should note that, in every case, the spectral change with time is a change in intensity ratio between the two distinct spectra of short-time and long-time fluorescences. Furthermore, overall fluorescence quantum yields of dimers and trimer are not so different from that of monomer with an exception of $o$-dimer; the relative quantum yields $\Phi_{\mathrm{F}}$, taken as 1.0 in monomer, are 0.91 in $p$-dimer, 0.80 in $m$-dimer and 0.48 in trimer [10]. This means that the ultrafast relaxation processes in two dimers and trimer are an energy conversion among the two excited states, without any significant energy dissipation. Namely, an intramolecular excitation energy transfer occurs in subpicosecond timescale from the higher energy state giving the fast fluorescence to the lowest energy state giving the slow fluorescence. In the case of $o$-dimer, however, $\Phi_{F}=0.1$ which means that an irreversible process of energy dissipation to environment is competing with the intramolecular energy transfer process.

The band positions of the absorption and the fast and slow fluorescence spectra are illustrated in Fig. 8 in an energy-level diagram where the two vibrational band energies of $(0,0)$ and $(1,0)$ in absorption and those of $(0,0)$ and $(0,1)$ 


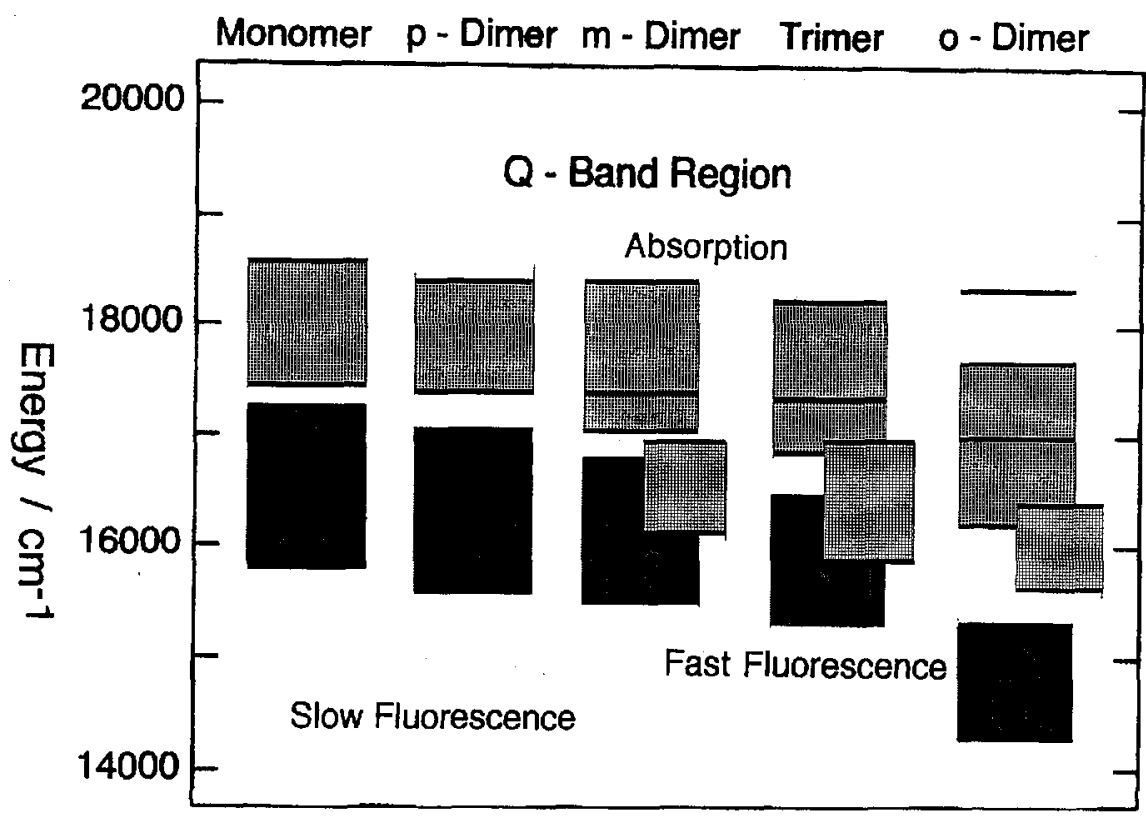

Fig. 8. Band positions of absorption and fast and slow fluorescence spectra. Horizontal lines show energy levels of vibrational bands $(0,0)$ and $(1,0)$ in absorption and $(0,0)$ and $(0,1)$ in fluorescence.

in fluorescence spectra are shown with horizontal lines. From the figure, we can recognize the mirror-image relationship and the Stokes shift between absorption and fluorescence spectra. For $m$ - and o-dimers and trimer, it can be seen that the band positions of the slow fluorescences correspond to those of the lowest lying absorption, and the fast fluorescence bands correspond to the second higher energy absorption bands. Let the two high and low energy levels denote as $S_{2}$ and $S_{1}$ states, respectively. To investigate quantitatively this relationship, energies of the band positions are summarized in Table IV, together with the Stokes shifts between $(0,0)$ bands in absorption and fluorescence. An important trend can be deduced with respect to vibrational band spacings and Stokes shifts.

In the absorption spectra, the vibrational band spacings between $(0,0)$ and $(1,0)$ are $1000-1100 \mathrm{~cm}^{-1}$ in $S_{2} \leftarrow S_{0}, 1100-1300 \mathrm{~cm}^{-1}$ in $S_{1} \leftarrow S_{0}$. In the fluorescence spectra, the vibrational spacing between $(0,0)$ and $(0,1)$ are $800-1100 \mathrm{~cm}^{-1}$ in $S_{2} \rightarrow S_{0}$ and $1100-1400 \mathrm{~cm}^{-1}$ in $S_{1} \rightarrow S_{0}$. The origins of these vibrations are to be totally symmetric vibrations of a $\mathrm{ZnP}$ ring with $1130-1580 \mathrm{~cm}^{-1}$ due to stretching vibration of $\mathrm{C}-\mathrm{C}, \mathrm{C}=\mathrm{C}$ and/or $\mathrm{C}-\mathrm{N}$ bonds, according to Raman spectroscopy $[18,19]$. One should note here that the vibrational spacing is fairly small in the fluorescence from $S_{2}$ relative to the $S_{1}$ emission. In the foregoing discussion (Sec. 3.2), we assume that the $S_{2}$ state is of local excitation and the excitation is localized at a $\mathrm{ZnP}$ ring. Then the $S_{2}$ fluorescence band profile could be similar to that of monomer. The experimental result that the vibrational band spacing of $S_{2}$ fluorescence differs from that of monomer or of the $S_{1}$ fluorescence can be explained in terms of a difference in the origins of two emissions. The fast $S_{2}$ flu- 
TABLE IV

Energy levels (in $\mathrm{cm}^{-1}$ ) of band maxima of absorption and fluorescence spectra.

\begin{tabular}{|c|c|c|c|c|c|}
\hline Bands & Monomer & $p$-Dimer & $m$-Dimer & Trimer & o-Dimer \\
\hline \multicolumn{6}{|l|}{ Absorption } \\
\hline \multirow[t]{3}{*}{ Soret band } & \multirow[t]{3}{*}{24270} & \multirow[t]{3}{*}{23529} & 24400 & 24250 & \multirow[t]{3}{*}{25000} \\
\hline & & & 23420 & 23350 & \\
\hline & & & & 22500 & \\
\hline$Q$ band & & & & & \\
\hline$S_{2} \leftarrow S_{0}(1,0)$ & - & - & 18403 & - & 18349 \\
\hline$(0,0)$ & - & - & 17391 & 17350 & 17301 \\
\hline$\Delta \nu$ & - & - & 1012 & - & 1048 \\
\hline$S_{1} \leftarrow S_{0}(1,0)$ & 18565 & 18416 & - & 18228 & 17794 \\
\hline$(0,0)$ & 17446 & 17361 & 17050 & 16950 & 16340 \\
\hline$\Delta \nu$ & 1119 & 1055 & - & 1278 & 1454 \\
\hline Fast fluorescence & & & & & \\
\hline$S_{2} \rightarrow S_{0}(0,0)$ & - & - & 16969 & 16949 & 16393 \\
\hline$(0,1)$ & - & - & 16126 & 15891 & 15625 \\
\hline$\Delta \nu$ & - & - & 843 & 1058 & 768 \\
\hline Slow fluorescence & & & & & \\
\hline$S_{1} \rightarrow S_{0}(0,0)$ & 17241 & 17007 & 16762 & 16667 & 15313 \\
\hline$(0,1)$ & 15798 & 15576 & 15504 & 15520 & 14265 \\
\hline$\Delta \nu$ & 1443 & 1431 & 1158 & 1147 & 1048 \\
\hline Stokes shifts & & & & & \\
\hline Fast fluorescence & - & - & 411 & 406 & 908 \\
\hline Slow fluorescence & 205 & 354 & 288 & 283 & 884 \\
\hline
\end{tabular}

orescence is a transition between highly excited vibrational levels of $S_{2}$ and $S_{0}$, the emitting level of which is isoenergetic to the levels generated by the internal conversion from the Soret state before thermal relaxation within $S_{2}$ state.

The experimental results mentioned above were obtained by excitation of the molecule into the Soret band. Similar ultrafast fluorescence decays were observed also with excitation into the $Q$ band in dimers and trimer. In this excitation, however, the precision of decay curve measurement is rather poor because of experimental difficulty; the wavelengths of excitation and monitored fluorescence become closer and the scattered light of laser makes it difficult to evaluate the relaxation rate. We consider, therefore, that the internal conversion from the Soret state to the $S_{2}$ state involved in the $Q$ band region takes place in a timescale less than $30 \mathrm{fs}$. Then the relaxation process that we observe here is the process from the $S_{2}$ state.in both the Soret band excitation and in the $Q$ band excitation. 
The present experimental results and discussion mentioned above leads to a conclusion. The ultrafast relaxation processes in $\mathrm{ZnP}$ dimers and trimer are ascribed to a process of excitation delocalization to entire molecular system due to an excitation transfer in a relatively strong interaction $\left(\sim 100 \mathrm{~cm}^{-1}\right)$, i.e., the medium coupling case in Förster's classification. The excitation delocalization takes place in timescale of 100-500 fs. The rate of this process is much faster in o-dimer $\left(1.3 \times 10^{13} \mathrm{~s}^{-1}\right)$ than in $m$-dimer $\left(1.9 \times 10^{12} \mathrm{~s}^{-1}\right)$ and trimer $\left(2.2 \times 10^{12} \mathrm{~s}^{-1}\right)$ which correspond to the magnitude of the interaction energy, $\sim 300 \mathrm{~cm}^{-1}$ in $o$-dimer and $\sim 100 \mathrm{~cm}^{-1}$ in $m$-dimer and trimer. It is worth noting that these timescales are comparable to those of the intramolecular vibrational redistribution and the vibrational relaxation in excited states of organic dye molecules in solution. Mokhtari, Chesnoy and Laubereau [5] demonstrated that Nile-blue in methanol exhibits $400 \mathrm{fs}$ IVR and $4 \mathrm{ps}$ VR. Martini and Hartland [6] demonstrated that hexamethyl indotricarbocyanine in organic solvents exhibits $300 \mathrm{fs}$ IVR and 1.7-7.1 ps VR, the latter of which depends on solvent; $1.2 \mathrm{ps}$ in methanol and $3.2 \mathrm{ps}$ in DMSO. These data allow us to consider that the excitation delocalization in $\mathrm{ZnP}$ dimers and trimer, particularly in o-dimer, occurs competing to or before the IVR and VR processes.

In recent studies on time-resolved spectroscopy of organic molecules in solution, increasing number of experimental data becomes available for kinetic parameters of photophysical processes from higher excited states. The present study may provide an additional example for the intramolecular relaxation process.

\section{References}

[1] Th. Förster, in: Modern Quantum Chemistry, Ed. O. Sinanoglu, Part III, Action of Light and Organic Crystals, Academic Press, New York 1965, p. 93.

[2] Th. Förster, in: Comparative Effects of Radiation, Eds. M. Burton, J.S. Kirby-Smith, J.L. Magee, Wiley, New York 1960, p. 300.

[3] V.M. Kenkre, R.S. Knox, Phys. Rev. Lett. 33, 803 (1974).

[4] R.M. Hochstrasser, R.B. Weisman, in: Radiationless Transitions, Ed. S.H. Lin, Academic Press, New York 1980 p. 317.

[5] A. Mokhtari, J. Chesnoy, A. Laubereau, Chem. Phys. Lett. 155, 593 (1989).

[6] I. Martini, G.V. Hartland, J. Phys. Chem. 100, 19764 (1996).

[7] Y. Jiang, G.J. Blanchard, J. Phys. Chem. 99, 7904 (1995).

[8] I. Yamazaki, N. Ohta, Pure Appl. Chem. 67, 209 (1995).

[9] I. Yamazaki, M. Yamaguchi, N. Okada, S. Akimoto, T. Yamazaki, N. Ohta, J. Lu$\min .72-74,71$ (1997).

[10] A. Osuka, B. Liu, K. Maruyama, J. Org. Chem. 58, 3582 (1993).

[11] A. Osuka, S. Nakajima, K. Maruyama, J. Org. Chem. 57, 366 (1992).

[12] S. Akimoto, S. Takaichi, T. Ogata, Y. Nishimura, I. Yamazaki, M. Mimuro, Chem. Phys. Lett. 260, 147 (1996).

[13] M. Mimuro, S. Akimoto, S. Takaichi, I. Yamazaki, J. Am. Chem. Soc. 119, 1452 (1997).

[14] W.T. Simpson, D.L. Paterson, J. Chem. Phys. 26, 588 (1957). 
[15] M. Kasha, Radiat. Res. 20, 55 (1963).

[16] A. Osuka, K. Maruyama, J. Am. Chem. Soc. 110, 4454 (1988).

[17] S. Eriksson, B. Källebring, S. Larsson, J. Mårtensson, O. Wennerström, Chem. Phys. 146, 165 (1990).

[18] R. Kumble, G.R. Loppnow, S. Hu, A. Mukherjee, M.A. Thompson, G.T. Spiro, J. Phys. Chem. 99, 5809 (1995).

[19] S. Hu, C.Y. Lin, M.E. Blackwood Jr, A. Mukherjee, T.G. Spiro, J. Phys. Chem. 99, 9694 (1995). 